\title{
LATTICE POINTS ON CIRCLES
}

\author{
JAVIER CILLERUELO
}

(Received 7 November 2000; revised 15 February 2001)

Communicated by W. W. L. Chen

\section{Abstract}

We prove that the lattice points on the circles $x^{2}+y^{2}=n$ are well distributed for most circles containing lattice points.

2000 Mathematics subject classification: primary $11 \mathrm{~N} 36$.

\section{Introduction}

The number of lattice points on the circle $x^{2}+y^{2}=n$ is denoted by $r(n)$. It is known that $r(n)$ is an unbounded function and it is a natural question to ask for the distribution of the $r(n)$ lattice points on the circle $x^{2}+y^{2}=n$.

In order to give a measure of that distribution, we consider the polygon with vertices at the $r(n)$ lattice points and denote by $S(n)$ the area of such a polygon. If the lattice points are well distributed, the area of the polygon must be close to the area of the circle, that is, $S(n) / \pi n \sim 1$.

If $r(n)>0$, trivially $2 / \pi \leq S(n) / \pi n<1$. In [1] we proved that the set $\{S(n) / \pi n$ : $r(n)>0\}$ is dense in the interval $[2 / \pi, 1]$. We also proved that $|S(n) / \pi n-1| \ll$ $(\log \log n / \log n)^{2}$ for infinitely many integers.

In this paper we prove that, in fact, for most integers $n$ such that $r(n)>0$, the quantity $S(n) / \pi n$ is close to 1 .

THEOREM 1.1. For any $n \leq x$ with $r(n)>0$,

$$
\frac{S(n)}{\pi n}>1-\left(\frac{11 \log \log \log x}{\log \log x}\right)^{2}
$$

I am indebted to Laura Fainsilber for calling my attention to this problem.

(C) 2002 Australian Mathematical Society $1446-7887 / 2002 \$ A 2.00+0.00$ 
with at most

$$
O\left(\frac{x}{(\log x)^{1 / 2} \log \log x \log \log \log x}\right)
$$

exceptions.

It should be noted that if we call $B_{x}=\{n \leq x: r(n)>0\}$, then $\left|B_{x}\right| \sim c x /(\log x)^{1 / 2}$.

\section{Background}

In the proof of Theorem 1.1 we will use the prime number theorem for Gaussian primes on angular sectors, and Selberg's sieve. We present them in a suitable form in this section.

THEOREM 2.1. Let $D$ be an angular sector of the circle $x^{2}+y^{2} \leq R^{2}$ with angle $\theta$. Then

$$
\sum_{\rho \in D} 1=\frac{\theta R^{2}}{\pi \log R}+O\left(\frac{R^{2}}{\log ^{2} R}\right)
$$

where $\rho=a+b i$ are primes in $Z[i]$ and the constant in the error term does not depend on $\theta$.

PROOF. Stronger versions of this result can be found in [2] and [3].

The sieving function $S(\mathscr{A}, P, z)$ denotes the number of terms of the sequence $\mathscr{A}$ that are not divisible by any prime $p \in P, p<z$. We denote by $\pi_{P}(x)$ the counting function of the sequence $P$.

THEOREM 2.2. If $P$ is an infinite subset of primes such that

$$
\begin{gathered}
\pi_{P}(x)=\alpha x / \log x+O\left(x / \log ^{2} x\right) \text { and } \mathscr{A}=\{1, \ldots, x\}, \text { then } \\
S(\mathscr{A}, P, x) \ll \frac{x}{(\log x)^{\alpha}} .
\end{gathered}
$$

PROOF. It will be a consequence of Selberg's sieve. For every square-free positive integer $d$, let $\left|A_{d}\right|$ denote the number of terms of the sequence $\mathscr{A}$ which are divisible by $d$. Then $\left|A_{d}\right|=x / d+r_{d}$, with $\left|r_{d}\right| \leq 1$. Let

$$
G(z)=\sum_{m<z, p \mid m \text { implies } p \in P} \frac{1}{m} .
$$


Selberg's sieve $[4$, page 180$]$ implies that

$$
S(A, P, z) \leq \frac{x}{G(z)}+\sum_{d<z^{2}, d \text { square-free }} 3^{\omega(d)} .
$$

Observe that

$$
G(z) \prod_{p<z, p \notin P}\left(1+\frac{1}{p}+\frac{1}{p^{2}}+\cdots\right) \geq \sum_{m<z} \frac{1}{m} \gg \log z
$$

and

$$
\prod_{p<z, p \notin P}\left(1+\frac{1}{p}+\frac{1}{p^{2}}+\cdots\right)=\prod_{p<z, p \notin P} \frac{p}{p-1} \leq \prod_{p} \frac{p^{2}}{p^{2}-1} \prod_{p<z, p \notin P}\left(1+\frac{1}{p}\right) .
$$

The first product is a constant and the second product can be estimated by taking logarithms:

$$
\log \left(\prod_{p<z, p \notin P}\left(1+\frac{1}{p}\right)\right) \leq \sum_{p<z, p \notin P} \frac{1}{p}=\sum_{p<z} \frac{1}{p}-\sum_{p<z, p \in P} \frac{1}{p} .
$$

The two sums can be handled using Abel's summation together with the formula

Then

$$
\pi(x)=\frac{x}{\log x}+O\left(\frac{x}{\log ^{2} x}\right), \quad \pi_{P}(x)=\alpha \frac{x}{\log x}+O\left(\frac{x}{\log ^{2} x}\right) .
$$

which yields

$$
\sum_{p<z} \frac{1}{p}-\sum_{p<z, p \in P} \frac{1}{p}=(1-\alpha) \log \log z+O(1)
$$

Observe that

$$
S(\mathscr{A}, P, z) \ll \frac{x}{(\log z)^{\alpha}}+\sum_{m<z^{2}, m \text { square -free }} 3^{\omega(m)} .
$$

$\sum_{m<z^{2}, m \text { square-free }} 3^{\omega(m)}=\sum_{m<z^{2}, m \text { square-free }}\left(2^{\omega(m)}\right)^{\log 3 / \log 2} \leq \sum_{m<z^{2}, m \text { square-free }} d^{2}(m) \ll z^{2} \log ^{3} z$.

Now if we choose $z=\left[x^{1 / 3}\right]$, we obtain $S(\mathscr{A}, P, x) \leq S(\mathscr{A}, P, z) \ll x /(\log x)^{\alpha}$.

Next, we will present two proposition needed to prove Theorem 1.1.

PROPOSITION 2.3. Let $\left\{x_{j}\right\}_{j=1}^{2 k}$ be a set of real numbers such that

$$
x_{j} \in I_{j}=\left(\frac{j-1}{2 k}, \frac{j}{2 k}\right], \quad j=1, \ldots, 2 k
$$

and for any real $\phi$ let $S=\left\{\phi+\sum_{j=1}^{2 k} \epsilon_{j} x_{j}, \epsilon_{j}= \pm 1\right\}$. Then, for any $j=1, \ldots k$, there exists $s \in S$ such that $\{s / 2\} \in J_{j}=((j-1) / k, j / k]$, where $\{s / 2\}$ denotes the fractional part of $s / 2$. 
ProOF. Let $\alpha=\phi-\sum_{j=1}^{2 k} x_{j}$. Then we can write

$$
S=\left\{\alpha+\sum_{j=1}^{2 k} \gamma_{j} x_{j}, \gamma_{j} \in\{0,2\}\right\} .
$$

The numbers $s_{i} / 2=\alpha / 2+x_{i}$, satisfy $s_{i+1} / 2-s_{i} / 2<1 / k$ for $i=1, \ldots, 2 k-1$ and $s_{1} / 2+1-s_{2 k} / 2<1 / k$. Then, for each interval $J_{j}$, there exists $s_{i} \in S$ such that $\left\{s_{i} / 2\right\} \in J_{j}$.

PROPOSITION 2.4. Let $n=n_{1} n_{2}$ such that $n_{j}=x_{j}^{2}+y_{j}^{2}, x_{j}+i y_{j}=\sqrt{n_{j}} e^{i \phi_{j}}$, $j=1,2$. Then, the angles $\pm \phi_{1} \pm \phi_{2}$ correspond to lattice points on the circle $x^{2}+y^{2}=n$.

PROOF. See [1] for more details.

\section{Proof of Theorem 1.1}

For each prime $p=2$ or $p \equiv 1(\bmod 4)$ let $\phi_{p}=(4 / \pi) \tan ^{-1}(a / b)$, where $a, b$ are the only integers such that $a^{2}+b^{2}=p, 0<a \leq b$. Then $\phi_{p} \in(0,1]$.

We split the interval $(0,1]$ in the $2 k$ intervals $I_{j}=((j-1) / 2 k, j / 2 k], j=1, \ldots, 2 k$ and we define the good numbers as

$$
G_{x}^{k}=\left\{n \in B_{x} ; n=p_{1} \cdots p_{2 k} m, \text { with } \phi_{p_{j}} \in I_{j}\right\},
$$

where we recall $B_{x}=\{n \leq x: \quad r(n)>0\}$.

In Proposition 3.1 we will prove that if $n \in G_{x}^{k}$, the lattice points on the circle $x^{2}+y^{2}=n$ are well distributed, and in Proposition 3.2 we will estimate the cardinality of the bad numbers, $B_{x}^{k}=B_{x} \backslash G_{x}^{k}$. Theorem 1.1 will be a consequence of these propositions for a suitable value of $k$.

PROPOSITION 3.1. If $n \in G_{x}^{k}$, then

$$
S(n) / \pi n>1-\pi^{2} / 6 k^{2} .
$$

PROOF. We can write $n=p_{1} \cdots p_{2 k} m$.

Obviously, $m$ has, at least, a representation as a sum of two squares, $m=a^{2}+b^{2}$, $a+i b=\sqrt{m} \exp (i(\pi / 4) \phi)$.

Proposition 2.4 implies that the angles $(\pi / 4)\left(\phi+\sum_{j=1}^{2 k-1} \epsilon_{j} \phi_{p_{j}}\right), \epsilon_{j}= \pm 1$ correspond to lattice points on the circle $x^{2}+y^{2}=n$. 
Suppose that $(\pi / 4) s$ is one of these angles. Then, due to the symmetry of the lattice, the angle $(\pi / 4) s-(\pi / 2)[s / 2]=(\pi / 2)\{s / 2\}$ also corresponds to a lattice point.

Now we apply Proposition 2.3 to conclude that for every $j=1, \ldots, k$ there exists an angle $s$ such that $\{s / 2\} \in J_{j}=((j-1) / k, j / k]$. In other words, for every $j=1, \ldots, k$ there exists a lattice point on the $\operatorname{arc} \sqrt{n} \exp (\pi \theta i / 2), \theta \in J_{j}$.

Again, due to the symmetry of the lattice we can find, for every $j=1, \ldots, k$ and for $r=0,1,2,3$, a lattice point on the $\operatorname{arc} \sqrt{n} \exp (\pi(\theta+r) i / 2), \theta \in J_{j}$.

Now let us choose a lattice point for each arc. Let $P_{0}$ be the polygon with vertices in these $4 k$ lattice points. Obviously, $S_{0}(n) \leq S(n)$, where $S_{0}(n)=\operatorname{Area}\left(P_{0}\right)$. Now we denote by $\theta_{1}, \ldots \theta_{4 k}$ the angles between each pair of two consecutive lattice points.

If we consider a sector with angle $\theta_{j}$ and radius $\sqrt{n}$, an easy geometric argument prove that the area of the part of the sector outside the triangle is $n\left(\theta_{j}-\sin \theta_{j}\right) / 2 \leq$ $n \theta_{j}^{3} / 12$. Then $\pi n-S_{0}(n) \leq(n / 12) \sum_{j=1}^{4 k} \theta_{j}^{3}$. We know that $\theta_{j} \leq \pi / k$ and that $\sum_{j=1}^{4 k} \theta_{j}=2 \pi$. Therefore, the maximum happens when the half of the angles are 0 and the other half are $\pi / k$. That is, $\pi n-S(n) \leq \pi n-S_{0}(n) \leq n \pi^{3} / 6 k^{2}$.

PROPOSITION 3.2. $\left|B_{x}^{k}\right| \ll k x /\left(\log ^{1 / 2+1 / 4 k} x\right)+k x^{3 / 4}$.

PROOF. If we apply Theorem 2.1 to the region

$$
D_{j}=\left\{(a, b): a^{2}+b^{2} \leq x, 0<a \leq b,(4 / \pi) \tan ^{-1}(a / b) \in I_{j}\right\}
$$

we obtain

$$
\pi_{P_{j}}(x)=\frac{x}{4 k \log x}+O\left(\frac{x}{\log ^{2} x}\right),
$$

where $P_{j}=\left\{p \not \equiv 3(\bmod 4): \phi_{p} \in I_{j}\right\}$.

On the other hand, if we denote by $Q=\{q \equiv 3(\bmod 4): q$ primes $\}$, the prime number theorem for arithmetic progressions says that $\pi_{Q}(x)=x /(2 \log x)+$ $O\left(x / \log ^{2} x\right)$. Then, if $Q_{j}=Q \cup P_{j}$ we obtain

$$
\pi_{Q_{j}}(x)=\left(\frac{1}{2}+\frac{1}{4 k}\right) \frac{x}{\log x}+O\left(\frac{x}{\log ^{2} x}\right) .
$$

We define, for any $1 \leq l \leq \sqrt{x}, \mathscr{A}_{l}=\left\{m \leq x / l^{2}\right\}$ and $\mathscr{A}_{l}^{*}=\left\{m \leq x / l^{2}\right.$ : $m$ square free\}. Now, suppose that $n \in B_{x}^{k}$ with $n=l^{2} m, m$ square free. Because $r(n)>0$, then $m$ has not prime divisors $q \equiv 3(\bmod 4)$. Since $n \notin G_{x}^{k}$, then there exists an integer $j$ such that $m$ has no prime divisors $p$ with $\phi_{p} \in I_{j}$. Then, that integer $n$ is shifted in $S\left(\mathscr{A}_{l}^{*}, Q_{j}, x / l^{2}\right)$. Thus,

$$
\left|B_{x}^{k}\right| \leq \sum_{1 \leq l \leq \sqrt{x}} \sum_{j=1}^{2 k} S\left(\mathscr{A}_{l}^{*}, Q_{j}, x / l^{2}\right) \leq \sum_{1 \leq l \leq \sqrt{x}} \sum_{j=1}^{2 k} S\left(\mathscr{A}_{l}, Q_{j}, x / l^{2}\right)
$$


For $l<x^{1 / 4}$ we apply Theorem 2.2 to each $S\left(\mathscr{A}_{l}, Q_{j}, x / l^{2}\right)$,

and then

$$
S\left(\mathscr{A}_{l}, Q_{j}, x / l^{2}\right) \ll \frac{x}{l^{2}\left(\log \left(x / l^{2}\right)\right)^{1 / 2+1 / 4 k}} \ll \frac{x}{l^{2}(\log x)^{1 / 2+1 / 4 k}}
$$

$$
\sum_{l \leq l \leq x^{1 / 4}} \sum_{j=1}^{2 k} S\left(\mathscr{A}_{l}, Q_{j}, x / l^{2}\right) \ll \frac{k x}{(\log x)^{1 / 2+1 / 4 k}}
$$

For $l \geq x^{1 / 4}$ we use the trivial estimate $S\left(\mathscr{A}_{l}, Q_{j}, x / l^{2}\right) \leq x / l^{2}$ and we obtain $\sum_{x^{1 / 4} \leq l} \sum_{j=1}^{2 k} S\left(\mathscr{A}_{l}, Q_{j}, x / l^{2}\right) \ll k x^{3 / 4}$.

To conclude Theorem 1.1 we apply Proposition 3.1 and Proposition 3.2 with $k=$ $[\log \log x /(8 \log \log \log x)]$. Observe that if $x$ is large enough, then

$$
k=[\log \log x /(8 \log \log \log x)]>\log \log x /((8.5) \log \log \log x) .
$$

Thus, for $n \in G_{x}^{k}$ and $x$ large enough,

$$
\frac{S(n)}{\pi n}>1-\frac{\pi^{2}}{6}\left(\frac{(8.5) \log \log \log x}{\log \log x}\right)^{2}>1-\left(\frac{11 \log \log \log x}{\log \log x}\right)^{2} .
$$

On the other hand,

$$
\begin{aligned}
\left|B_{k}(x)\right| & \ll \frac{\log \log x}{\log \log \log x} \frac{x}{(\log x)^{1 / 2}(\log x)^{(2 \log \log \log x) /(\log \log x)}} \\
& \ll \frac{x}{(\log x)^{1 / 2} \log \log x \log \log \log x} .
\end{aligned}
$$

\section{References}

[1] J. Cilleruelo, 'The distribution of the lattice points on circles', J. Number Theory 43 (1993), 198-202.

[2] I. Kubilyus, 'The distribution of Gaussian primes in sectors and contours', Leningrad. Gos. Univ. Uchen. Zap. Ser. Mat. Nauk 137 (1950), 40-52.

[3] T. Mitsui, 'Generalized prime number theorem', Japan J. Math. 26 (1956), 1-42.

[4] M. Nathanson, Additive number theory: the classical bases (Springer, New York, 1996).

\section{Departamento de Matemáticas}

Universidad Autónoma de Madrid

28049 Madrid

Spain

e-mail: franciscojavier.cilleruelo@uam.es 